\title{
Linear Pulse-Coupled Oscillators Model-A New Approach for Time Synchronization in Wireless Sensor Networks
}

\author{
Zhulin An ${ }^{1,2}$, Hongsong $\mathrm{Zhu}^{1,3}$, Meilin Zhang ${ }^{1}$, Chaonong $\mathrm{Xu}^{4}$, Yongjun $\mathrm{Xu}^{1}$, Xiaowei $\mathrm{Li}^{1}$ \\ ${ }^{1}$ Institute of Computing Technology, Chinese Academy of Sciences, Beijing, China \\ ${ }^{2}$ Graduate University of Chinese Academy of Sciences, Beijing, China \\ ${ }^{3}$ Institute of Software, Chinese Academy of Sciences, Beijing, China \\ ${ }^{4}$ Department of Computer Science and Technology, China University of Petroleum-Beijing, Beijing, China \\ Email: \{anzhulin,xyj,lxw\}@ict.ac.cn, zhuhongsong@is.iscas.ac.cn,meilin.zhang1988@gmail.com,xuchaonong@cup.edu.cn \\ Received November 13, 2009; revised December 14, 2009; accepted December 7, 2009
}

\begin{abstract}
Mutual synchronization is a ubiquitous phenomenon that exists in various natural systems. The individual participants in this process can be modeled as oscillators, which interact by discrete pulses. In this paper, we analyze the synchronization condition of two- and multi-oscillators system, and propose a linear pulse-coupled oscillators model. We prove that the proposed model can achieve synchronization for almost all conditions. Numerical simulations are also included to investigate how different model parameters affect the synchronization. We also discuss the implementation of the model as a new approach for time synchronization in wireless sensor networks.
\end{abstract}

Keywords: Synchronization, Biologically Inspired Algorithms, Pulse-Coupled Oscillators, Wireless Sensor Networks

\section{Introduction}

Synchronous flashing of fireflies is a fascinating phenomenon that a large number of scientists have been drawn to research on. The mechanism behind this phenomenon has been investigated for nearly a century. In 1915, Blair observed and tried to examine the scientific reason behind it [1]. He analogize firefly to electric battery-each flash temporarily exhausts the battery, and a period of recuperation is required before the next flash can be emitted. The flash of a leader stimulates the discharge of others, and in the end this makes all the fireflies flash in concert. Richmond presented a similar postulation that if one firefly is ready to flash and sees flashes of others, it starts sooner than otherwise [2]. In 1988, Buck summarized these two battery-analogy mechanisms, and proposed the phase-advanced model. He defined "late sensitivity window" which is a time interval during the period between a firefly's flashings, and concluded that when a photic stimulus (flashing) occurs during the late sensitivity window, it initiates an immediate flash and resets the status of the firefly.

Although the phase-advanced model gives a fine explanation to certain varieties of fireflies' synchronization behavior, the interaction, which is usually called coupling, between fireflies is narrowly limited to late sensitivity window. Peskin extended coupling to any time of the cycle. In his book published in 1975 [3], Peskin proposed a more detailed pulse-coupled oscillators model for the natural pacemaker of a human heart. He modeled a pacemaker as a system consisting of mutual coupled "integrate-and-fire" oscillators. Each oscillator is characterized by state $x$, which satisfies

$$
\frac{d x}{d t}=-\gamma x+S_{0} \quad 0 \leq x \leq 1
$$

where $\gamma$ and $S_{0}$ are intrinsic properties of the oscillators. When $x=1$ an oscillator fires then jumps back to $x=0$, and the states of the other oscillators will be kicked up by coupling strength $\varepsilon$. Through his research, Peskin found that due to coupling, the states of the oscillators tend to come to the same. And as the system evolves, all oscillators would eventually achieve the state of discharging in steps. Peskin proved that for a two oscillators system with small $\varepsilon$ and $\gamma$, the system approaches a state in which the oscillators are firing synchronously. Mirollo and Strogatz extended Peskin's 
work, and proved that an N-oscillators system with non-linear dynamics will achieve synchronization for almost all conditions [4].

The models discussed above were all based on pulsecoupling. That is, the oscillators interacted with each other only when one of them fires. In 1975, Kuramoto presented a phase-coupling model [5]. In Kuramoto model, the dynamic of oscillator $i$ in an N-oscillators system can be described as

$$
\frac{d \theta_{i}}{d t}=\omega_{i}+\frac{K}{N} \sum_{j=1}^{N}\left(\sin \theta_{j}-\sin \theta_{i}\right)
$$

where $\omega_{i}$ is the natural frequency of oscillator $i, K$ is the coupling strength. Kuramoto proved numerically that as the coupling strength is increased above a critical value, the system exhibits a spontaneous transition from incoherence to collective synchronization despite the difference in the natural frequencies of the oscillators [6]. The analytical results of Kuramoto model were obtained by Crawford ten years later. Using center manifold theory, Crawford calculated the weakly nonlinear behavior of the infinite-dimensional system in the neighborhood of the incoherent state. A comprehensive review can be found in [7].

When reviewing the development of studies in synchronous flashing of firefly, it can be observed that the main researchers ranged from biologists to mathematicians and physicians, then to computer scientists and engineers. Recently, the application of the mechanism in synchronization of computer network and neural network makes the research of pulse-coupled oscillators again a popular topic. When applying oscillator based methods to network synchronization, phase-coupling is not ideal, because the coupling during all oscillating cycle is difficult to be implemented. However, the pulse-coupling models proposed by Peskin and Mirollo \& Strogatz are not suitable for direct application either, because there are certain assumptions in the model that are difficult to be guaranteed in practical applications. Firstly, those models are all based on instant coupling, implying the pulse is received without any delay, while the propagation delay in wireless communication cannot be neglected. Ernst, Pawelzik, et al. [8,9] presented a complete mathematical analysis of two oscillator system with delay, and numerical simulation of multi-oscillators. They came to the conclusion that the synchronization can still be achieved if inhibitory couplings $(\varepsilon<0)$ are adopted. Secondly, all-to-all coupling limits the application in computer networks which are by nature distributed systems. A comprehensive summary of works on Mirollo and Strogatz's model (M\&S model) with neighbor communication can be found in [10]. There is also work reported for the application of the pulse-coupled model. Hong and Scaglione firstly implemented the M\&S model on a Ultra Wideband network [11], and in [12] they comprehensively investigated how the parameters in pulse-coupled model affected the synchronization precision. Werner-Allen, Tewari, et al. [13] discussed their encounter problems when implementing the model on a wireless sensor network (WSN) testbed, and proposed some programming technologies to overcome them.

From the above discussion, we know that the application of pulse-coupled model to the synchronization of wireless network is no easy work. Moreover, when this model is applied to WSN, which usually adopts a microcontroller as its processor, the limitation of computational ability must also be considered. The non-linear dynamic makes it difficult for a micro-controller to work efficiently. (e.g. Ref. [13] used first order Taylor expansion for approximation.) In this paper, we propose a pulse-coupled oscillators model with linear dynamic. The synchronization issue is discussed, and we prove that the presented model can achieve synchronization for almost all conditions. We also include numerical simulations to validate the effectiveness of the model and investigate how model parameters affect the synchronization.

The rest of the paper is organized as follows. Section 2 describes the model and coupling among oscillators. In Section 3 and 4 respectively, we prove two- and multicoupled oscillators can achieve synchronization for the presented model. Section 5 presents numerical simulation and analysis of the results. In Section 6, we summarize our major work, and discuss the implementation of the model as a new approach for time synchronization in wireless sensor networks.

\section{Model Descriptions}

For the Peskin model $\frac{d x}{d t}=-\gamma x+S_{0}$, let $\gamma=0$, $S_{0}=\frac{1}{T}$ and $t \in[0, T]$, we have $\frac{d x}{d t}=\frac{1}{T}$. Integrating the differential equation above yields $x=\frac{t}{T}$. We define $T$ as the cycle period and $\phi=\frac{t}{T}$ as the phase variable. Then we obtain our linear model

$$
x=f(\phi)=\phi \quad \phi \in[0,1]
$$

Due to the fact that the state variable always equals to the phase variable, we use $\phi$ to represent both the state variable and the phase variable.

Coupling is an important mechanism. It is the only communication method among oscillators. Therefore, a multi-oscillator system can be modeled as an "integrate-and-fire" oscillator network. Each oscillator in the system evolves following linear relationship mentioned in (3). When $\phi_{i}=1$, the $i$ th oscillator "fires", and returns to the state $\phi_{i}=0$. At the same time, it pulls all the 
other oscillators up by its coupling strength, or pulls them up to firing, whichever is less. That is,

$$
\phi_{i}=1 \Rightarrow \phi_{j}^{\prime}=\min \left(1, \phi_{j}+\varepsilon_{i}\right), \quad \forall j \neq i
$$

where $\varepsilon_{i}$ is the coupling strength of $i$. We assume that all the oscillators' coupling strength stay constant and are distributed in a close interval $[a, b]$.

\section{Proof of Synchronization of Two Coupled Oscillators}

An oscillators system consisting of two coupled oscillators is the simplest, and hence can be studied thoroughly. Therefore, we first discuss the synchronization of two coupled oscillators. We define and compute the firing map and return map, based on which we present the synchronization condition of two oscillators. Then we prove that, if the condition is satisfied, the two oscillators will always achieve synchronization.

\subsection{Firing Map and Return Map of Two Coupled Oscillators}

Firing map and return map are effective tools for study of the evolution process of oscillators system. Snapshots are taken when an oscillator fires, and by studying these snapshots we can explore the relationship of oscillators phases.

Definition 1 [return map of B about A]: Given two oscillators $\mathrm{A}$ and $\mathrm{B}$, assuming that at the instant after one firing of $\mathrm{A}$ the phase of $\mathrm{B}$ is $\phi$, the return map of $\mathrm{B}$ about $\mathrm{A} R_{B \mid A}(\phi)$ is defined as the phase of $\mathrm{B}$ after the next firing of A.

Definition 2 [firing map of A about B]: Given two oscillators $\mathrm{A}$ and $\mathrm{B}$, assumes that at the instant after one firing of $\mathrm{A}$ the phase of $\mathrm{B}$ is $\phi$, the firing map of $\mathrm{A}$ about $\mathrm{B} h_{A \mid B}(\phi)$ is defined as the phase of $\mathrm{A}$ after the next firing of $\mathrm{B}$.

For oscillators A and B, assume at the instant after A fires, the phase of $\mathrm{B}$ is $\phi$. After a time period of $1-\phi$, $B$ reaches its firing threshold. At the same time the phase of A changes from zero to $1-\phi$. B fires after an instant, and $A$ jumps to $\phi_{A}=1-\phi+\varepsilon_{B}$ or 1 , whichever is less. If $\phi_{A}=1$, the two oscillators achieve synchronization; therefore we assume that $\phi_{A}=1-\phi+\varepsilon_{B}<1$, we have the firing map of A about B

$$
h_{A \mid B}(\phi)=1-\phi+\varepsilon_{B}
$$

From the analysis above, after one firing, the system has evolved from the initial state $\left(\phi_{A}, \phi_{B}\right)=(0, \phi)$ to the current state $\left(\phi_{A}, \phi_{B}\right)=\left(h_{A \mid B}(\phi), 0\right)$. This implies the system is similar as what it was at the beginning, with $\phi$ being replaced by $h_{A \mid B}(\phi)$ and two oscillators being interchanged. Therefore, the return map of B about A can be calculated as

$$
R_{B \mid A}(\phi)=h_{B \mid A}\left(h_{A \mid B}(\phi)\right)=\phi+\left(\varepsilon_{A}-\varepsilon_{B}\right)
$$

\subsection{Synchronization Condition of Two Coupled Oscillators}

From (6), it can be established that each time when A fires, the phase of $\mathrm{B}$ increases by $\varepsilon_{A}-\varepsilon_{B}$ from the last firing of $\mathrm{A}$. With this fact, we can infer the following synchronization condition for two coupled oscillators.

Theorem 1 [synchronization condition of two coupled oscillators]: Given two oscillators A and B with their coupling strengths satisfying

$$
\varepsilon_{A} \neq \varepsilon_{B}
$$

they will achieve synchronization.

Proof: From our assumption, we know that $\varepsilon_{A}$ and $\varepsilon_{B}$ maintain constant during the evolution. Hence, since $\varepsilon_{A} \neq \varepsilon_{B} \Leftrightarrow \varepsilon_{A}-\varepsilon_{B} \neq 0$, we obtain

$$
\begin{array}{lll}
R_{B \mid A}\left(\phi_{B}\right)<\phi_{B} & \text { if } \varepsilon_{A}-\varepsilon_{B}<0 \\
R_{B \mid A}\left(\phi_{B}\right)>\phi_{B} & \text { if } & \varepsilon_{A}-\varepsilon_{B}>0
\end{array}
$$

Therefore, from any initial state of $\mathrm{A}$ and $\mathrm{B}$, the phases of the two oscillators move monotonically toward 0 or 1 . In other words, the two coupled oscillators will always reach synchronization.

\section{Proof of Synchronization of Multi-Oscillators System}

The evolution of a multi-oscillators system is much more complicated than that of two coupled oscillators. When two oscillators fires synchronously, they will clump together, and absorb to a group that acts as one single oscillator with a bigger coupling strength. This makes it easier for other oscillators to join their group, and leads to a positive feedback process. There may exist several groups during the evolution, but as this process goes on, the number of groups decreases, and eventually, all groups will clump to one big group, when the whole system achieves synchronization.

As with the discussion of two coupled oscillators, we first define firing map and return map of multi-oscillators system, and then discuss the absorption, through which the oscillators clump together into groups. Base on these definitions, we present the synchronization condition for a multi-oscillators system. Finally, we prove that the synchronization condition can be satisfied, except for a set of coupling strengths with zero Lebesgue measure. 


\subsection{Firing Map, Return Map and Absorption in Multi-Oscillators System}

Firing map and return map are also essential in discussing synchronization in multi-oscillators system. Consider two oscillators $i$ and $j$ in an $\mathrm{N}$ oscillators system that never synchronize with other oscillators in the system during their evolution. Assume the phase of $j$ is $\phi$, at the instant that $i$ has just fired. Without considering the firings of other oscillators, after $1-\phi, j$ will fire. However, the firings of other oscillators decrease this period to $1-\phi-\sum_{k \in \Omega} \varepsilon_{k}$ ( $\Omega$ is the set of subscripts of oscillators which will fire before $j$ fires). Similarly, the firings of oscillators in $\Omega$ also increase the phase of $j$ by $\sum_{k \in \Omega} \varepsilon_{k}$.

Hence, we have the firing map of $i$ about $j$ :

$$
h_{i \mid j}(\phi)=1-\phi-\sum_{k \in \Omega} \varepsilon_{k}+\varepsilon_{j}+\sum_{k \in \Omega} \varepsilon_{k}=1-\phi+\varepsilon_{j}
$$

Similar to the case of two coupled oscillators, the return map of $i$ about $j$ can be written as

$$
R_{i \mid j}(\phi)=\phi+\left(\varepsilon_{j}-\varepsilon_{i}\right)
$$

When two oscillators synchronized, they will clump together and form a synchronous firing group which acts as a single oscillator with larger coupling strength. If that happens we call an absorption occurred, and the coupling strength of a group formed by A and B can be computed as

$$
\varepsilon_{A B}=\varepsilon_{A}+\varepsilon_{B}
$$

\subsection{Synchronization Condition of Multi-Oscillators System}

Similar to the discussion of two coupled oscillators, our analysis of multi-oscillators system is also based on the return map. The difference is when discussing two oscillators in a multi-oscillator, the firing of other oscillators must also be considered.

Theorem 2 [synchronization condition of multi-oscillators system]: Given an $\mathrm{N}$ oscillators system, let $S=\left\{\varepsilon_{1}, \varepsilon_{2}, \cdots, \varepsilon_{N}\right\}$ be the set of the coupling strengths of all oscillators in the system. The system will achieve synchronization, if the following conditions are satisfied.

$$
\sum_{\varepsilon_{m} \in S_{1}} \varepsilon_{m} \neq \sum_{\varepsilon_{n} \in S_{2}} \varepsilon_{n} \quad \forall S_{1}, S_{2} \subset S, \quad S_{1} \cap S_{2}=\varnothing
$$

Proof: First, we are to prove by contradiction that if the condition is satisfied, absorption is sure to occur. Assume absorption never occurs during the evolution of an $\mathrm{N}$ oscillators system. For two individual oscillators or oscillator groups $i, j$ in the system, let

$$
\varepsilon_{i}=\sum_{\varepsilon_{m} \in S_{1}} \varepsilon_{m}, \quad \varepsilon_{j}=\sum_{\varepsilon_{n} \in S_{2}} \varepsilon_{n}
$$

Since $\varepsilon_{i}$ and $\varepsilon_{j}$ are the sums of several $\varepsilon \mathrm{s}$ and none of the oscillators in $i$ and $j$ are identical, we have

$$
S_{1}, S_{2} \subset S, \quad S_{1} \cap S_{2}=\varnothing
$$

From (11), we know that

$$
\varepsilon_{i} \neq \varepsilon_{j}
$$

Furthermore, for a multi-oscillator in which absorption never occurs, $\varepsilon_{i}$ and $\varepsilon_{j}$ stay constant. Therefore, similar to the discussion in the case of two coupled oscillators, from the return map (9) we know the phases of $i, j$ are driven monotonically toward $\phi=0$ or $\phi=1$. That is to say, absorption must occur, which contradicts with our assumption. Therefore, absorption in an $\mathrm{N}$ oscillators system always occurs.

From the analysis above, we know that absorption always occurs in a multi-oscillators system satisfying condition (11). And after the absorption, an $\mathrm{N}$ oscillators system evolves to an $\mathrm{N}-1$ oscillators system with a slightly different set of parameters. As this process continues, all $\mathrm{N}$ oscillators will eventually evolve into one single group, and the synchronization of the whole system is achieved.

We now prove the synchronization condition (11) can be satisfied except for a set of coupling strengths with zero measure.

Theorem 3: For an $\mathrm{N}$ oscillators system, each oscillator in the system has a coupling strength within $[a, b]$. The system will achieve synchronization, except for a set of coupling strengths in $[a, b]^{N}$ with zero Lebesgue measure.

Proof: Let $\mathrm{E}=\left(\varepsilon_{1}, \varepsilon_{2}, \cdots, \varepsilon_{n}\right)$, which is an element in an $\mathrm{N}$-dimensions subset $[a, b]^{N}$ of $R^{n}$, and $S=\left\{\varepsilon_{1}, \varepsilon_{2}, \cdots, \varepsilon_{N}\right\}$ be a set consisting of all the components of $\mathrm{E}$. We are now going to prove the set of $\mathrm{E}$ in $[a, b]^{N}$ which satisfies

$$
\sum_{\varepsilon_{m} \in S_{1}} \varepsilon_{m}=\sum_{\varepsilon_{n} \in S_{2}} \varepsilon_{n} \exists S_{1}, S_{2} \subset S, \quad S_{1} \cap S_{2}=\varnothing
$$

has a Lebesgue measure of zero.

Let $f(\mathrm{E})=\sum_{\varepsilon_{m} \in S_{1}} \varepsilon_{m}-\sum_{\varepsilon_{n} \in S_{2}} \varepsilon_{n}$, then $f(E)$ can be represented as

$$
f(\mathrm{E})=a_{1} \varepsilon_{1}+a_{2} \varepsilon_{2}+\cdots+a_{N} \varepsilon_{N}
$$

where $\quad a_{k}= \begin{cases}1 & \varepsilon_{k} \in S_{1} \\ 0 & \varepsilon_{k} \in S-S_{1}-S_{2} \quad \text {,which indicates } \\ -1 & \varepsilon_{k} \in S_{2}\end{cases}$ $f(\mathrm{E})$ is a hyperplane in $[a, b]^{N}$. Furthermore, the amount of such hyperplanes is less than $3^{N} / 2$, not 
unlimited. Therefore, the set of $\mathrm{E}$ satisfying condition (12) has the a Lebesgue measure of zero.

With Theorem 2, we found the synchronization condition for a multi-oscillators system, and proved if the condition is satisfied the system will achieve synchronization. Then in Theorem 3, we proved the condition will be satisfied except for a set of coupling strengths with zero measure. Combining the two theorems, we proved the presented multi-oscillators system will achieve synchronization except for a set of coupling strengths with zero measures.

\section{Numerical Simulation and Analysis}

To validate that the synchronization can be established for the presented model and investigate how model parameters affect the synchronization process, we perform a numerical simulation of the model in a Java environment. Every simulation consists of an initialization stage and a simulation stage. In the former, the parameters of the model are initialized, which includes the number of oscillators $(n)$, the period $(T)$, oscillator phase $(\phi)$ and coupling strength $(\varepsilon)$. Due to the limitation of computer

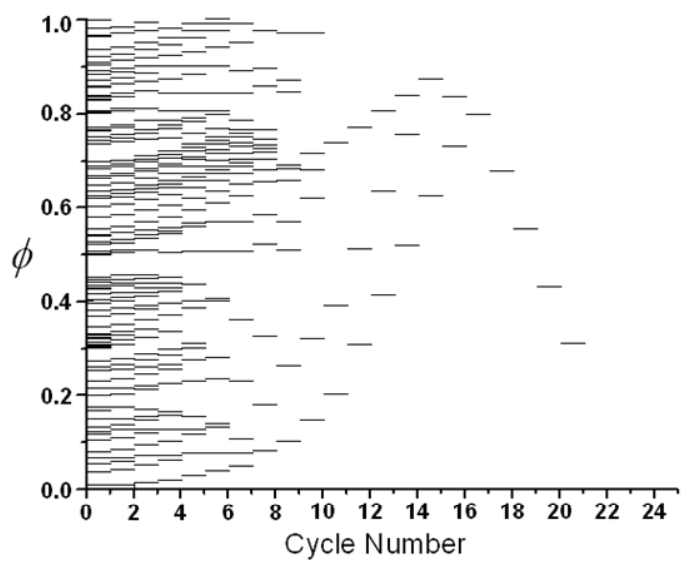

(a)

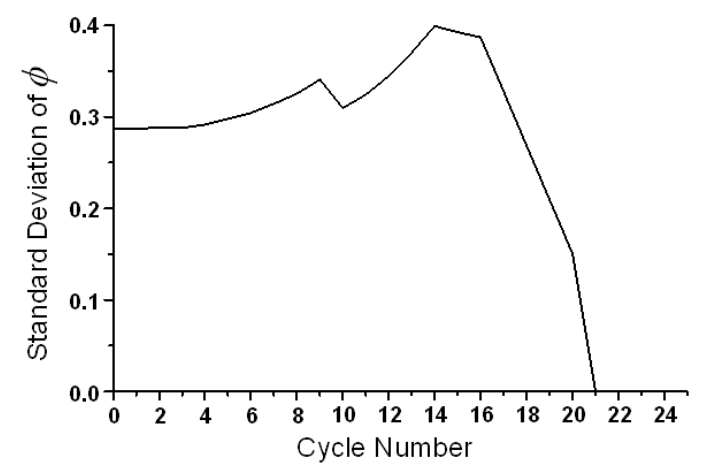

(b)

Figure 1. Phase and standard deviation of phase during the synchronization process with $\mathrm{n}=100, \varepsilon_{-}$base $=\mathbf{0 . 0 0 5}$, $\varepsilon \_$ratio $=0.1$.

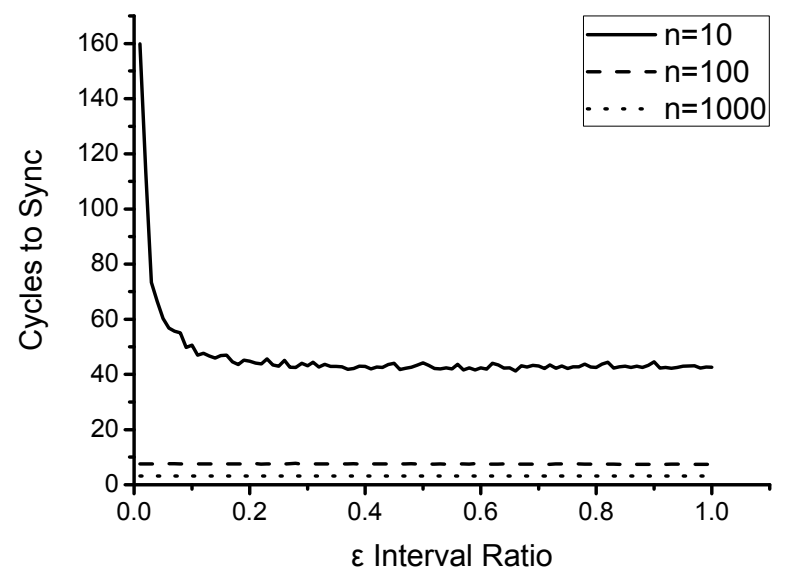

Figure 2. Cycle numbers to achieve synchronization versus $\varepsilon \_$ratio with $\varepsilon_{-}$base $=0.01$ for $n=10,100$ and 1000 .

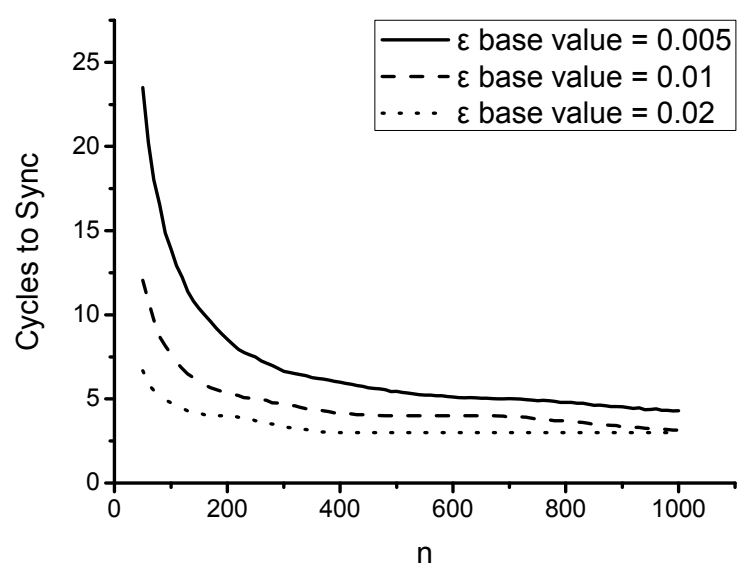

Figure 3. Cycle numbers to achieve synchronization versus $\mathrm{n}$ with $\varepsilon_{\_}$ratio $=0.1$ for various $\varepsilon_{-}$base. Because when $\mathbf{n}$ and $\varepsilon$ base are both very small, the cycle numbers until synchronization is going to be very large. To show the detail of all the plots, the figure is ploted from $\mathbf{n}=\mathbf{5 0}$.

simulation, $T, \phi$ and $\varepsilon$ are all discretized to integers. Specifically, $T$ is set to a large number (10000000), and $\phi$ is generated randomly between [0, $T$ ]. $\varepsilon$ is generated randomly between [ $\varepsilon_{-}$base $-\frac{1}{2} \varepsilon_{-}$base $e^{*} \varepsilon_{-}$ratio, $\varepsilon_{-}$base $+\frac{1}{2} \varepsilon_{-}$base $e^{*} \varepsilon_{-}$ratio ], where $\varepsilon_{-}$base , $\varepsilon_{-}$ratio are "coupling strength base value" and "coupling strength interval ratio" respectively. The simulation stage consists of many cycles. During each cycle, the oscillator with the maximum phase is found first, and the system is forwarded to the firing instant of the oscillator. Then all the oscillators' phases are adjusted according to the coupling strength of the fired oscillator. Finally, all the fired oscillators are combined into a group with new coupling strength computed by (10). This cycle repeats until there is only one group left. 
Additionally, to avoid the effect of arbitrary randomness of $\phi$ and $\varepsilon$, every simulation related to cycles to synchronization is done 1000 times, and the average of 800 values in the center range (eliminate the maximum 100 results and the minimum 100 results) is adopted as the final result.

We first simulate the synchronization process. The results are shown in Figure 1. Figure 1(a) shows the phases of oscillators at different cycles. Each dash in the figure represents the phase of a particular oscillator or oscillator group, and the phases are plotted every time when the phase of oscillator No.0 returns to 0 . (As a result, there is always a dash at $\phi=0$ ) We can see from the plot that as cycles continue, the number of dashes decrease, indicating that the oscillators gradually clump into groups. In the end, when there is only one group left, the oscillators achieve synchronization. Figure 1(b) shows the standard deviation of the oscillators' phases during the same process. From the figure, we can find that at the beginning the standard deviation generally increases as the evolution progresses, but each time when absorption happens the standard deviation decreases. Near the end, when there are only two groups, the standard deviation decreases dramatically, and finally reaches zero.

We then investigate how the parameters $\left(n, \varepsilon_{-}\right.$base and $\varepsilon_{-}$ratio ) affect the number of cycles needed to achieve synchronization. Figure 2 shows required cycle number to achieve synchronization as a function of $\varepsilon_{\text {_ratio }}$ with $\varepsilon_{\text {_base }}=0.01$ for $n=10,100$ and 1000. From Figure 2, we can see that when $n$ is big enough the cycle number to synchronize does not change with $\varepsilon_{-}$ratio. We now discuss the reason behind this phenomenon. Suppose $i$ is an oscillator in a multi-oscillators system, then every time when $i$ fires, its phase increases by $\sum_{k=1, k \neq i}^{n} \varepsilon_{k}$. In this simulation, although $\varepsilon_{-}$ratio varies, the sum of all $\varepsilon$ lies on $\varepsilon_{-}$base. Furthermore when $n$ is big enough, the sum $\sum_{k=1, k \neq i}^{n} \varepsilon_{k}$ will approximate the sum of all $\varepsilon$. Therefore, the cycles needed stay the same. We also notice that when $n$ and $\varepsilon_{-}$ratio are both small, more cycles are needed to synchronize. This is because, if $\varepsilon_{-}$ratio is small the $\varepsilon$ of all oscillators will be almost the same. Due to the linear dynamic, the deviation of all the oscillators' phases increases slowly, so more cycles are needed.

Following above analysis, we know that the $\varepsilon_{-}$ratio will not affect the result if $n$ is not a very small number. So we fix the value of $\varepsilon_{-}$ratio to 0.1 and discuss how number of cycles needed to synchronize varies with different values of $n$ and $\varepsilon_{-}$base.

Figure 3 shows number of cycles required to achieve

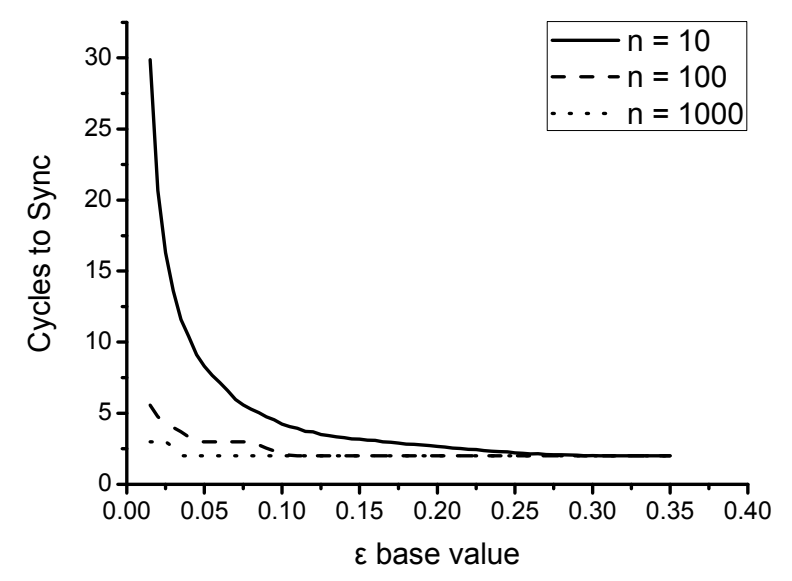

Figure 4. Cycle numbers to achieve synchronization versus $\varepsilon_{-}$base with $\varepsilon_{-}$ratio $=0.1$ for various $n$. Because when $\mathbf{n}$ and $\varepsilon$ _base are both too small, the cycle number to synchronized is too large. To show the detail of all the plots, the figure is plotted from $\varepsilon_{-}$base $=\mathbf{0 . 0 1 5}$.

synchronization versus $n$ for various $\varepsilon_{-}$base. For a fixed $\varepsilon_{-}$base, cycle numbers decrease with the increase of $n$. The reason is that the more oscillators there are in the system, the easier it is for the oscillators to absorb to synchronous firing groups. And the $\varepsilon$ of a group is the sum of $\varepsilon$ of all oscillators in that group, so it is equivalent to increase the $\varepsilon$ of the oscillator. Therefore, system tends to synchronize earlier.

Figure 4 shows cycle numbers to achieve synchronization versus $\varepsilon_{-}$base with $\varepsilon_{-}$ratio $=0.1$ for various values of $n$. In the figure, we can find that for a certain number of oscillators, the larger $\varepsilon$ is, the less cycles are needed to achieve synchronization. This is because the return map increases with the increase of $\varepsilon$, and the system tends to synchronize faster.

From the simulation result presented in this section, we can draw the following conclusion. First, as we discussed in Subsection 4.1, with the evolution of a muti-oscillator system, the oscillators in the system tend to clump together into synchronous firing groups. When there is only one group left, the system achieves synchronization. Second, the number of cycles to achieve synchronization depends on $n$ and $\varepsilon_{-}$base; a larger $n$ or $\varepsilon_{-}$base may lead to faster synchronization, and $\varepsilon_{-}$ratio will have no effect on the number of cycles to synchronize, unless $n$ is very small. To summarize, the simulations match well with our theoretical analysis.

\section{Conclusions and Future Work}

In this paper, we proposed a model for linear pulse-coupled oscillators system with different coupling strengths. We discussed the synchronization condition for both 
two- and multi-oscillators system, and proved that the proposed system can achieve synchronization for almost all conditions. Simulations of the model in a Java environment are also included, which validated the model and investigated how different parameters affect the synchronization.

As a swarm of fireflies, a WSN consists of a number of wireless sensor nodes that interact with each other via radio communications. Therefore, if the model presented in this paper is applied as a new approach for time synchronization in WSNs, the algorithm would be more scalable and robust. In the implement, the phase described in the model is represented by a counter, which moves monotonically towards a threshold $T$, corresponding to the period of the oscillator. When the counter reaches $T$, it jump back to zero and triggers an interrupt follow with a new cycle. In the interrupt handler, a packet containing the node's coupling strength $\varepsilon$ is sent out, which will be used by other nodes to add to their own counter. In this manner, all the counters will be synchronized after a few cycles as what has been discussed in the simulation. However, there is also factors must be considered before this model can be adopted practically, including the message delay, the message collision, the network topology and so on. The implement of the model on a WSN testbed will be included in our future work.

\section{Acknowledgement}

The research presented in this paper was supported in part by National Natural Science Foundation of China (NSFC) under grants No.(60772070, 60873244, 60633060, 60831160526), in part by High-Tech Research and Development Program of China (863) under grants No.(2007AA12Z321, 2007AA01Z113), and in part by National Basic Research Program of China (973) under grant No.(2005CB321604, 2006CB303000). Authors also wish to acknowledge help of Sen $\mathrm{Yu}$ in writing the English version of this paper.

\section{References}

[1] K. G. Blair, "Luminous Insects," Nature, Vol. 96, No. 2406, pp. 411-415, 1915.
[2] C. A. Richmond, "Fireflies flashing in unison," Science, Vol. 71, No. 1847, pp. 537-538, 1930.

[3] C. S. Peskin, "Self-synchronization of the cardiac pacemaker," in Mathematical Aspects of Heart Physiology, New York University: New York. pp. 268-278, 1975.

[4] R. E. Mirollo and S. H. Strogatz, "Synchronization of pulse-coupled biological oscillators," SIAM Journal on Applied Mathematics, Vol. 50, No. 6, pp. 1645-1662, 1990.

[5] Y. Kuramoto, "Self-entrainment of a population of coupled non-linear oscillators," in International Symposium on Mathematical Problems in Theoretical Physics, pp. 420-422, 1975.

[6] Y. Kuramoto, "Chemical oscillations, waves, and turbulence," Springer Series in Synergetics, Berlin, Springer-Verlag, pp. 164, 1984.

[7] S. H. Strogatz, "From kuramoto to crawford: exploring the onset of synchronization in populations of coupled oscillators," Physica D: Nonlinear Phenomena, Vol. 143, No.1-4, pp. 1-20, 2000.

[8] U. Ernst, K. Pawelzik, and T. Geisel, "Synchronization induced by temporal delays in pulse-coupled oscillators," Physical Review Letters, Vol. 74, No. 9, pp. 1570-1573, 1995.

[9] U. Ernst, K. Pawelzik, and T. Geisel, "Delay-induced multistable synchronization of biological oscillators," Physical Review E, Vol. 57, No. 2, pp. 2150-2162, 1998.

[10] O. Simeone, et al. "Distributed synchronization in wireless networks." IEEE Signal Processing Magazine, Vol. 25, No. 5, pp. 81-97, 2008.

[11] Y. W. Hong and A. Scaglione, "Time synchronization and reach-back communications with pulse-coupled oscillators for UWB wireless ad hoc networks," in Proceedings of IEEE Conference on Ultra Wideband Systems and Technologies, 2003.

[12] Y. W. Hong, and A. Scaglione, "A scalable synchronization protocol for large scale sensor networks and its applications," IEEE Journal on Selected Areas in Communications, Vol. 23, No. 5, pp. 1085-1099, 2005.

[13] G. Werner-Allen, et al. "Firefly-inspired sensor network synchronicity with realistic radio effects," in Proceedings of the 3rd international conference on Embedded networked sensor systems. ACM: San Diego, California, USA. pp. 142-153, 2005. 